\begin{tabular}{c|c|c|} 
SELECCIONES MATEMÁTICAS \\
Universidad Nacional de Trujillo \\
ISSN: $2411-1783$ (Online) \\
2021; Vol. 8(1): $1-11$.
\end{tabular}

\title{
A comparative analysis of methods: mimetics, finite differences and finite elements for 1-dimensional stationary problems
}

\section{Un análisis comparativo de los métodos: miméticos, diferencias finitas y elementos finitos para problemas estacionarios 1-dimensional}

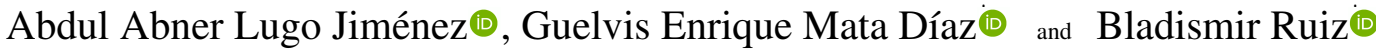 \\ Received, Set. 17, 2020 \\ Accepted, Jun. 01, 2021
}

How to cite this article:

Lugo Jiménez AA. et al. A comparative analysis of methods: mimetics, finite differences and finite elements for 1-dimensional stationary problems. Selecciones Matemáticas. 2021;8(1):1-11. http://dx.doi.org/10.

17268 /sel.mat.2021.01.01

\begin{abstract}
Numerical methods are useful for solving differential equations that model physical problems, for example, heat transfer, fluid dynamics, wave propagation, among others; especially when these cannot be solved by means of exact analysis techniques, since such problems present complex geometries, boundary or initial conditions, or involve non-linear differential equations. Currently, the number of problems that are modeled with partial differential equations are diverse and these must be addressed numerically, so that the results obtained are more in line with reality. In this work, a comparison of the classical numerical methods such as: the finite difference method (FDM) and the finite element method (FEM), with a modern technique of discretization called the mimetic method (MIM), or mimetic finite difference method or compatible method, is approached. With this comparison we try to conclude about the efficiency, order of convergence of these methods. Our analysis is based on a model problem with a one-dimensional boundary value, that is, we will study convection-diffusion equations in a stationary regime, with different variations in the gradient, diffusive coefficient and convective velocity.
\end{abstract}

Keywords . Mimetic method, finite element method, finite difference method, conservative methods, convergence.

\section{Resumen}

Los métodos numéricos son útiles para resolver ecuaciones diferenciales que modelan problemas físicos, por ejemplo, transferencia de calor, dinámica de fluidos, propagación de ondas, etc.; en especial cuando estos no pueden ser resueltos por medio de técnicas de análisis exacto, ya que dichos problemas presentan complejas geometrías, condiciones de contorno o iniciales, o bien involucran ecuaciones diferenciales no lineales. En la actualidad, la cantidad de problemas que se modelan con ecuaciones diferenciales parciales son diversos y estos deben ser abordados numéricamente, para que los resultados obtenidos se ajusten más a la realidad. En este trabajo se aborda una comparación de los métodos numéricos clásicos como lo son: el método de diferencias finitas (FDM) y el método de los elementos finitos (FEM), con una técnica moderna de discretización llamado el método mimético (MIM), o método de diferencias finitas miméticas o método compatible. Con esta comparación se trata de concluir sobre la eficiencia, orden de convergencia de estos métodos. Nuestro análisis está basado en un problema modelo con valor de frontera unidimensionales, es decir, estudiaremos ecuaciones de convección-difusión en régimen estacionario, con variaciones distintas en el gradiente, coeficiente difusivo y velocidad convectiva.

Palabras clave. Método mimético, método de los elementos finitos, método de diferencias finitas, métodos conservativos, convergencia.

\footnotetext{
* Instituto Superior de Formación Docente Salomé Ureña, Recinto Félix Evaristo Mejía. Santo Domingo, República Dominicana. (abdul. lugo@isfodosu. edu.do).

$\dagger$ Universidad de Los Andes, Facultad de Ciencias. Mérida, Venezuela. (gmata@ula.ve).

‡Universidad Técnica de Manabí, Instituto de Ciencias Básicas. Portoviejo, Ecuador. (bladismir@ula.ve).
} 
1. Introducción. Los métodos numéricos se utilizan generalmente para la aproximación numérica de soluciones de diversos problemas, que se derivan de aplicaciones tales como ingeniería, ciencias básicas entre otras. La mayoría de estos problemas se modelan a partir de ecuaciones diferenciales parciales. Para aproximar su solución, los métodos numéricos más usados son el método de diferencias finitas (FDM) y el método de elementos finitos (FEM). Sin embargo, en las últimas décadas, un nuevo esquema numérico conservativo basado en diferencias finitas conocido como método mimético (MIM) [1, 2, 3, 4, 5], ha mostrado su superioridad al esquema de diferencias finitas tradicionales ver [6, 7].

Cada uno de estos métodos presenta ventajas para ciertos tipos de problemas y desventajas en otros. A pesar de esto, y sin introducir ningún tipo de sesgo, el FEM ha predominado en casi todas las aplicaciones que resultan de interés científico hoy en día. Y, en aquellas donde el FDM mantuvo su preponderancia, el FEM estándar ha sufrido modificaciones para optimizar su aplicabilidad en estos problemas. Por ejemplo, se puede citar a [8,9] para problemas de dinámica de fluidos y [10] en el caso de problemas con conductividad discontinua. Por otro lado, mucho se ha dicho sobre la eficiencia de los métodos miméticos en comparación con el FDM [11, 12], principalmente en problemas difusivos estacionarios. Sin embargo, se ha dejado de lado su comparación con el FEM, justificando el hecho a una alta complejidad de la implementación computacional del mismo.

En este trabajo, buscando una mejor interpretación de las diferencias que existen entre los tres métodos, se presenta un análisis numérico de las propiedades que posee cada uno de estos métodos: convergencia, precisión en la frontera del dominio, eficiencia en diversos problemas, flexibilidad al dominio físico e implementación. Dicho análisis se enmarca dentro de la ecuación de convección-difusión estacionaria para problemas unidimensionales; se busca facilitar el análisis de los métodos, dejando de lado, momentáneamente, las dificultades analíticas y geométricas que implica un dominio físico 2D.

El resto del artículo se estructura de la siguiente manera. En el siguiente apartado se introduce el problema de valor de frontera usado junto a sus condiciones de frontera (Robin). En el tercer apartado se describen brevemente los métodos numéricos; posteriormente, en la cuarta sección, se realiza la experimentación numérica y la discusión de los resultados. Finalmente, se presentan las conclusiones obtenidas y las referencias del trabajo.

2. Problema Modelo. La ecuación que modela la transferencia de calor por convección-difusión en régimen estacionario en su forma más simple (problema unidimensional con coeficiente de difusión y velocidad de convección constantes) viene dada por

$$
k(x) \frac{d^{2}}{d x^{2}} u(x)+\nu(x) \frac{d}{d x} u(x)=f(x), \quad \text { en } \quad \Omega=(a, b),
$$

donde $u(x)$ representa la temperatura (variable del problema) en un punto $x$ del dominio $\Omega=(a, b) ; k>0$, el coeficiente de difusión térmica; $\nu$, la velocidad convectiva (o advectiva) y $f$, una función escalar que describe la existencia de una fuente o sumidero en el problema. La ecuación se completa al definir las condiciones de frontera:

$$
\alpha_{a} u(a)+\beta_{a} \frac{d}{d x} u(a)=\gamma_{a} \quad, \quad \alpha_{b} u(b)+\beta_{b} \frac{d}{d x} u(b)=\gamma_{b},
$$

con $\left\{\alpha_{i}, \beta_{i}, \gamma_{i}\right\}, i=a, b$, parámetros reales conocidos, y dependiendo de su valor se tendrán condiciones de contorno Dirichlet, Neumann o Robin. Aunque la solución general del problema de valor de frontera (2.1)(2.2) se puede obtener en gran parte de las configuraciones del problema mediante métodos analíticos, el mismo resultará apropiado para dejar ver las propiedades de los métodos numéricos en estudio, y simplificar la visualización y análisis de los resultados.

3. Métodos Numéricos. En este apartado se describen brevemente los tres métodos utilizados. Una descripción detallada del esquema mimético a ser utilizado en este artículo puede ser vista, entre otras, en $[1,12]$. Para el caso del FEM existe un sin número de referencias que introducen el método desde distintas vertientes; aquí, nos inclinamos por [13, 14, 15]. Por último, en el caso de FDM se remite al lector a [16].

3.1. Método Mimético. Los métodos miméticos se basan en la discretización de los operadores clásicos de las EDP (divergencia, gradiente, laplaciano y rotacional) de tal forma que ellos satisfagan una versión discreta del Teorema de Stokes o identidad de Green:

$$
\langle\mathbf{D} v, f\rangle_{Q}+\langle v, \mathbf{G} f\rangle_{P}=\langle\mathbf{B} v, f\rangle_{I} .
$$

Aquí, D, G y B son las versiones discretas de sus continuos correspondientes: gradiente $(\nabla)$, divergencia $(\nabla \cdot)$ y operador de frontera $\partial / \partial n$. Los \langle\rangle representan un producto interior generalizado con pesos $Q, P$ e 
I. Usando la identidad (3.1) se obtiene una relación para el operador de frontera

$$
\mathbf{B}=Q \mathbf{D}+\mathbf{G}^{\mathbf{t}} P .
$$

Para la discretización, se define una malla cuya geometría está dada por los nodos $x_{i}$, con $i=0,1, \ldots, N$, y las celdas de la misma son $\operatorname{los}$ intervalos $\left[x_{i-1}, x_{i}\right]$, para $i=1, \ldots, N$. El tamaño de la celda, $h$, viene dado por $h=1 / N$, suponiendo que la malla está distribuida uniformemente. Los nodos intermedios de las celdas queda dado por $x_{i+1 / 2}=\left(x_{i}+x_{i+1}\right) / 2$, ver Figura 3.1. El operador divergencia se definen en el centro de las celdas, la solución se define en los nodos de la frontera y en los centros de cada una de las celdas, mientras el operador gradiente en los nodos $x_{i}$ que definen las celdas (ver Figura 3.1).

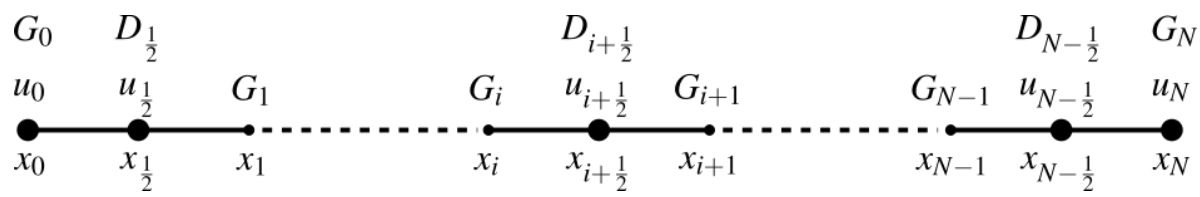

Figura 3.1: Malla escalonada unidimensional.

En este trabajo, se analiza el método mimético que surge de los operadores discretos de segundo orden (tanto en frontera como en los nodos internos) introducidos por [2] y dados en (3.3) y (3.4).

$$
\begin{gathered}
\vec{G} \vec{u} \equiv\left[\begin{array}{c}
(G u)_{0} \\
(G u)_{1} \\
\vdots \\
(G u)_{N-1} \\
(G u)_{N}
\end{array}\right]=\frac{1}{h}\left[\begin{array}{cccccc}
-\frac{8}{3} & 3 & -\frac{1}{3} & 0 & \cdots & 0 \\
0 & -1 & 1 & 0 & \cdots & 0 \\
\vdots & \ddots & \ddots & \ddots & \ddots & \vdots \\
0 & \cdots & 0 & -1 & 1 & 0 \\
0 & \cdots & 0 & \frac{1}{3} & -3 & \frac{8}{3}
\end{array}\right]\left[\begin{array}{c}
u_{0} \\
u_{\frac{1}{2}} \\
\vdots \\
u_{N-\frac{1}{2}} \\
u_{N}
\end{array}\right] \\
\vec{D} \vec{v} \equiv\left[\begin{array}{c}
0 \\
D_{\frac{1}{2}} v \\
D_{\frac{3}{2}} v \\
\vdots \\
D_{N-\frac{3}{2}} v \\
D_{N-\frac{1}{2}} v \\
0
\end{array}\right]=\frac{1}{h}\left[\begin{array}{cccccc}
0 & 0 & 0 & 0 & \cdots & 0 \\
-1 & 1 & 0 & 0 & \cdots & 0 \\
0 & -1 & 1 & 0 & \cdots & 0 \\
\vdots & \ddots & \ddots & \ddots & \ddots & \vdots \\
0 & \cdots & 0 & -1 & 1 & 0 \\
0 & \cdots & 0 & 0 & -1 & 1 \\
0 & \cdots & 0 & 0 & 0 & 0
\end{array}\right]\left[\begin{array}{c}
v_{0} \\
v_{1} \\
v_{2} \\
\vdots \\
v_{N-2} \\
v_{N-1} \\
v_{N}
\end{array}\right]
\end{gathered}
$$

En (3.4) aparecen dos filas de ceros (una en la parte superior y otra en la parte inferior), con esto se busca obtener una matriz cuadrada al componer operadores. El operador de frontera $\mathbf{B}$ queda dado explícitamente por

$$
\mathbf{B}=\left[\begin{array}{ccccccc}
-1 & 0 & 0 & \cdots & 0 & 0 & 0 \\
1 / 8 & -1 / 8 & 0 & \cdots & 0 & 0 & 0 \\
-1 / 8 & 1 / 8 & 0 & \cdots & 0 & 0 & 0 \\
0 & 0 & 0 & \cdots & 0 & 0 & 0 \\
\vdots & \vdots & \vdots & \ddots & \vdots & \vdots & \vdots \\
0 & 0 & 0 & \cdots & 0 & 0 & 0 \\
0 & 0 & 0 & \cdots & 0 & -1 / 8 & 1 / 8 \\
0 & 0 & 0 & \cdots & 0 & 1 / 8 & -1 / 8 \\
0 & 0 & 0 & \cdots & 0 & 0 & 1
\end{array}\right]
$$


A partir de las discretizaciones de los operadores, la aproximación mimética para la ecuación de convección-difusión (2.1) queda dada por

$$
k \mathbf{D G} U+\nu \mathbf{G} U=(k \mathbf{D G}+\nu \mathbf{G}) U=F,
$$

donde $U$ representa la solución aproximada mimética a la solución exacta, $u$, del problema

$$
U=\left(U\left(x_{0}\right), U\left(x_{1 / 2}\right), \ldots, U\left(x_{N-1 / 2}\right), U\left(x_{N}\right)\right)^{\mathrm{t}},
$$

y $F$ representa la restricción de $f$ a la malla mimética:

$$
F=\left(f\left(x_{0}\right), f\left(x_{1 / 2}\right), \ldots, f\left(x_{N-1 / 2}\right), f\left(x_{N}\right)\right)^{\mathrm{t}}
$$

Como el operador divergencia discretizado no actúa sobre la frontera, entonces las condiciones de contorno Robin (2.2) se obtienen a partir

$$
[[\alpha]+[\beta](\mathbf{B G})] U=f_{b},
$$

donde el vector $f_{b}$ resulta de restringir el término no homogéneo de las condiciones de contorno a la malla escalonada, es decir, $f_{b}=\left(\gamma_{a}, 0, \ldots, 0, \gamma_{b}\right)^{\mathrm{t}}$. Las matrices $[\alpha]$ y $[\beta]$ son tales que $\alpha_{1,1}=\alpha_{a}, \alpha_{N+2, N+2}=$ $\alpha_{b}, \beta_{1,1}=\beta_{a}, \beta_{N+2, N+2}=\beta_{b}$, y el resto de entradas son cero.

A partir de (3.5) y (3.6), el esquema mimético para la ecuación de convección-difusión (2.1) sujeto a las condiciones de contorno Robin (2.2) queda dado por

$$
[[\alpha]+[\beta](\mathbf{B G})+k \mathbf{D G}+\nu \mathbf{G}] U=F+f_{b} .
$$

3.2. Método de diferencias finitas. En el método de diferencias finitas los operadores diferenciales presentes en la ecuación diferencial son discretizados (aproximados) para los nodos de la malla, $x_{i}$, con $i=0,1, \ldots, N$, usando esquemas en diferencia que provienen del truncamiento del polinomio de Taylor (ver Figura 3.2).

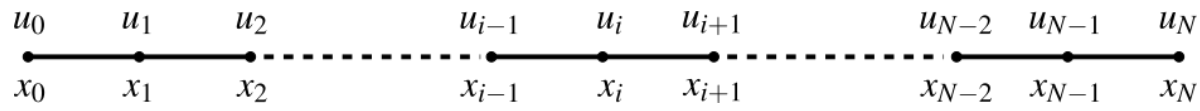

Figura 3.2: Malla unidimensional para el método de diferencias finitas.

Para obtener el mismo orden de convergencia que el esquema mimético (3.5) planteado en el apartado anterior, se propone un método usando los esquemas de diferencia de segundo orden:

$$
\begin{aligned}
\frac{d^{2}}{d x^{2}} u\left(x_{i}\right) & =\frac{u\left(x_{i+1}\right)-2 u\left(x_{i}\right)+u\left(x_{i-1}\right)}{h^{2}}+\mathcal{O}\left(h^{2}\right) \\
\frac{d}{d x} u\left(x_{i}\right) & =\frac{u\left(x_{i+1}\right)-u\left(x_{i-1}\right)}{2 h}+\mathcal{O}\left(h^{2}\right) .
\end{aligned}
$$

En los nodos interiores de la malla, la discretización realizada a partir del esquema de segundo orden es riguroso. No obstante, al usar el esquema de diferencias centradas de segundo orden para discretizar las condiciones de frontera de Robin (2.2) se emplean nodos fantasmas, lo cual hace al método poco riguroso.

El esquema en diferencias finitas para la ecuación de convección-difusión (2.1) queda dado entonces

$$
\left\{\begin{array}{l}
{\left[\left(k_{0}-\nu_{0} \frac{h}{2}\right) \frac{2 h \alpha_{a}}{\beta_{a}}-2 k_{0}\right] U_{0}+2 k_{0} U_{1}=h^{2} f_{0}+\left(k_{0}-\nu_{0} \frac{h}{2}\right) \frac{2 h}{\beta_{a}} \gamma_{a},} \\
\left(k_{i}-\nu_{i} \frac{h}{2}\right) U_{i-1}-2 k_{i} U_{i}+\left(k_{i}+\nu_{i} \frac{h}{2}\right) U_{i+1}=h^{2} f_{i}, \quad \text { para } i=1: N-1, \\
2 k_{N} U_{N-1}-\left[\left(k_{N}+\nu_{N} \frac{h}{2}\right) \frac{2 h \alpha_{b}}{\beta_{b}}+2 k_{N}\right] U_{N}=h^{2} f_{N}-\left(k_{N}+\nu_{N} \frac{h}{2}\right) \frac{2 h}{\beta_{b}} \gamma_{b},
\end{array}\right.
$$

donde $U_{i}$, con $i=0,1, \ldots, N$, define la solución aproximada del problema. Los subíndices en el esquema denotan la evaluación en los nodos $x_{i}$ de la malla de diferencias finitas (por ejemplo, $f_{i}=f\left(x_{i}\right)$ ). 
3.3. Método de los elementos finitos. En el FEM se tiene una solución $u_{\mathrm{H}}:=\sum a_{i} \phi_{i}(x)$, con $a_{i}$ coeficientes a determinar, y $\phi_{i}$ funciones base de un espacio de dimensión finita $\mathscr{V}_{\mathrm{H}} \subset \mathscr{V}$, con $\mathscr{V}$ el espacio de funciones admisibles de la forma débil o variacional del problema

$$
B(u, v)=l(v), \quad \forall v \in \mathscr{V},
$$

donde

$$
B(u, v)=\theta_{a} \alpha_{a} u(a)-\theta_{b} \alpha_{b} u(b)-\int_{a}^{b}\left[(k v)^{\prime}+\nu v\right] u^{\prime} d x
$$

es una forma bilineal y

$$
l(v)=\int_{a}^{b} f v d x-\theta_{b} \gamma_{b}+\theta_{a} \gamma_{a}
$$

es un funcional lineal. En (3.10) y (3.11), $\theta_{a}$ y $\theta_{b}$ quedan dadas por $\theta_{a}=k(a) v(a) / \beta_{a}$ y $\theta_{b}=k(b) v(b) / \beta_{b}$, y $\mathscr{V}$ el espacio de Sobolev $\mathbf{H}^{1}(\Omega)$. Los coeficientes $a_{i}$ son determinados a partir del sistema de ecuaciones que surge al sustituir $u_{\mathrm{H}}$ en la forma variacional (3.9).

Es conocido a partir del Lema de Ceá [17] que el error cometido en la aproximación de una solución exacta mediante elementos finitos viene acotada por el error de aproximación. Ahora bien, dado que el error de aproximación depende directamente del tamaño de los elementos, cuanto mayor sea el número de elementos menor será el error de aproximación.

4. Resultados Numéricos. En este apartado, se presentan y analizan los resultados numéricos obtenidos (comparativamente) por los métodos propuestos en distintas configuraciones del problemas (2.1)-(2.2) en el dominio $\Omega=(0,1)$. Para el análisis se usan las normas del máximo, $\|\cdot\|_{\infty}$, y la norma $L_{2}$ definidas por definidas por

$$
\|e\|_{\infty}:=\operatorname{máx}\left\{e_{j}:=\left|U_{j}-u_{j}\right|\right\}, \quad\|e\|_{2}^{2}:=\sum_{j} H_{j+1 / 2}^{-1}\left|U_{j}-u_{j}\right|^{2},
$$

con $j=i+1 / 2$, y $i=0, \ldots, N-1$.

4.1. Experimentación \#1. Se resuelve (2.1)-(2.2) con coeficiente de difusión $k=1$, velocidad convectiva $\nu=0$, condiciones de contorno Robin:

$$
\begin{aligned}
& \alpha u(0)-u^{\prime}(0)=-20 /\left(e^{20}-1\right), \\
& \alpha u(1)+u^{\prime}(1)=0,
\end{aligned}
$$

con $\alpha:=-20 e^{20} /\left(e^{20}-1\right)$ y un término fuente, $f(x)$, definido tal que la solución analítica del problema queda dada por $u(x)=\left(e^{20 x}-1\right) /\left(e^{20}-1\right)$. La Figura 4.1 muestra las soluciones aproximadas junto a la solución analítica del problema en una malla uniforme de 20 puntos.



Figura 4.1: Experimentación \#1: Comparación de las soluciones aproximadas en un malla de 20 nodos. En la frontera, el FEM supera en precisión a los otros dos esquemas.

La ampliación en la frontera derecha muestra la mejor precisión del FEM al imponer condiciones de frontera, seguido del esquema mimético que supera a FDM, aunque en esta se esté usando esquemas 
de segundo orden para aproximar la frontera (ver (3.8)). Esta propiedad de aproximación se mantiene al aumentar el número de nodos y se repite para cualquier problema con coeficientes $k$ y $\nu$ constantes. La igualdad en la precisión de los nodos de frontera es solo alcanzada cuando se tienen condiciones de frontera Dirichlet.

La Figura 4.2 muestra los errores alcanzados (a la izquierda medidos a partir de la norma del máximo, $\|\cdot\|_{\infty} \mathrm{y}$, a la derecha usando norma $L_{2},\|\cdot\|_{2}$ ). En la gráfica, las pendientes de las rectas definen el orden de convergencia de los métodos y mientras más abajo se encuentre, mejor será el esquema que represente.


Figura 4.2: Experimentación \#1 Errores numéricos en la norma $\|\cdot\|_{\infty}$ (izquierda) y la norma $\|\cdot\|_{2}($ derecha).

\begin{tabular}{|c|ccc|ccc|}
\hline \multirow{2}{*}{$\begin{array}{c}\text { \# de } \\
\text { Elementos }\end{array}$} & \multicolumn{3}{|c|}{ Norma $\|\cdot\|_{\infty}$} & \multicolumn{3}{c|}{ Norma $\|\cdot\|_{2}$} \\
\cline { 2 - 7 } & FDM & MIM & FEM & FDM & MIM & FEM \\
\hline 1000 & 1.999944 & 2.016795 & 4.000306 & 0.986027 & 1.000097 & 1.999880 \\
3000 & 1.999990 & 2.007299 & 4.022705 & 0.994014 & 1.000020 & 1.999978 \\
5000 & 1.999998 & 2.003188 & 3.485599 & 0.997404 & 1.000004 & 1.999996 \\
7000 & 2.000000 & 2.002136 & -10.558 & 0.998307 & 1.000002 & 1.999967 \\
9000 & 1.999999 & 2.001412 & 15.251 & 0.998740 & 1.000001 & 2.000043 \\
11000 & 1.999998 & 2.001592 & -27.111 & 0.998995 & 1.000001 & 2.000664 \\
\hline
\end{tabular}

Tabla 4.1: Experimentación \#1: Orden de convergencia en Norma $\|\cdot\|_{\infty}$ y Norma $\|\cdot\|_{2}$.

Los métodos alcanzan el orden de convergencia teórico en la norma del máximo (ver Cuadro 4.1). Sin embargo, en norma del máximo, para este problema, el FEM presenta una superconvergencia alcanzando el $\varepsilon$ de máquina. Por tal motivo, las oscilaciones que presenta la gráfica para el caso del FEM es debido a errores de redondeo de máquina y no a pérdida de precisión del FEM. Esta característica no se mantiene en otras configuraciones del problema como se verá posteriormente. En norma $L_{2}$, el FEM presenta un orden dos en su convergencia (como era de esperar, ver [14]), mientras los otros dos métodos presentan un orden uno de convergencia (Figura 4.2 y la tabla 4.1 ).

4.2. Experimentación \#2. En este caso, se define el problema modelo (2.1)-(2.2) con coeficiente de difusión $k(x)=1 / \alpha+\alpha\left(x-x_{0}\right)^{2}$, velocidad convectiva $\nu(x)=k^{\prime}(x)$, con $\alpha=250$ y $x_{0}=0,75$, condiciones de contorno Robin:

$$
\begin{aligned}
& u(0)+u^{\prime}(0)=\alpha /\left(1+\alpha^{2} x_{0}^{2}\right), \\
& u(1)+u^{\prime}(1)=-\arctan \left(\alpha\left(1-x_{0}\right)\right)-\arctan \left(\alpha x_{0}\right),
\end{aligned}
$$

y un término fuente, $f(x)$, definido tal que la solución analítica del problema viene dada por $u(x)=$ $(1-x)\left[\arctan \left(\alpha\left(x-x_{0}\right)\right)+\arctan \left(\alpha x_{0}\right)\right]$. La Figura 4.3 (izquierda) muestra las soluciones aproximadas junto a la solución analítica del problema para una malla uniforme de 60 puntos. A la derecha, se ilustra la convergencia asintótica, $h \rightarrow 0$, de las aproximaciones en el nodo de frontera $x=1$. Cuando la discretización es gruesa (pocos nodos) el método mimético supera levemente al FEM. El método FDM necesita superar los 1500 elementos para alcanzar la precisión de los otros dos métodos. 

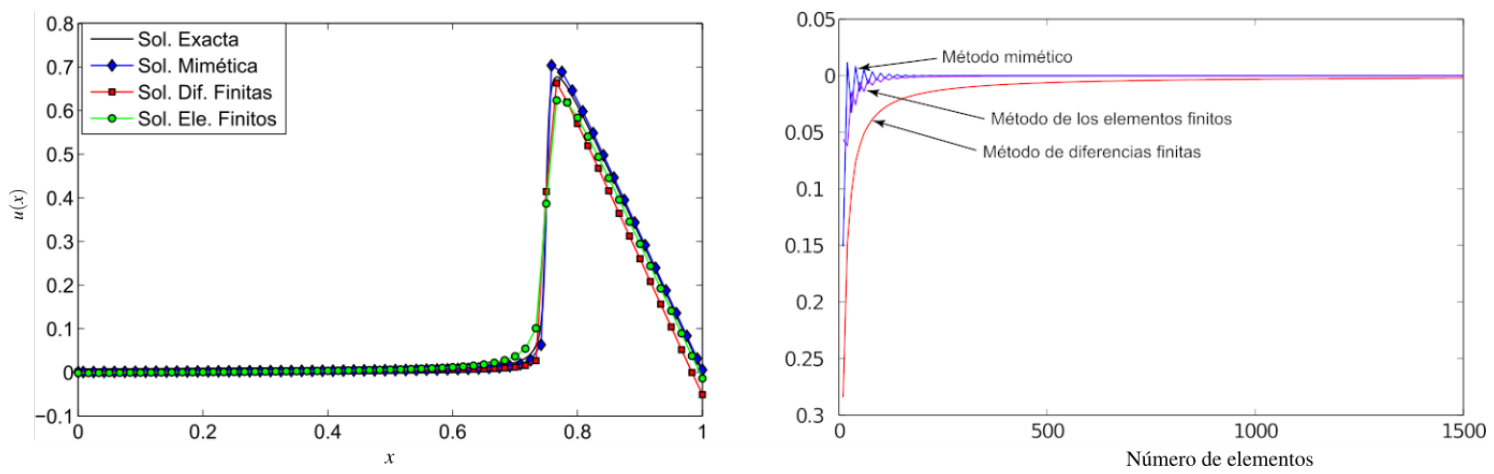

Figura 4.3: Experimentación \#2: Soluciones aproximadas y exactas en una cuadrícula de 60 nodos (izquierda). Convergencia de los métodos en el nodo de frontera $\mathrm{x}=1$ al aumentar el número de nodos en la malla (derecha)

Para este ejemplo, se puede ver que, $u(0)=u(1)=0$, lo cual simplifica las condiciones de frontera y, por lo tanto, la formulación variacional del problema (ver, ecuaciones (3.10)-(3.11)). En estos casos, la mejor precisión en los nodos de frontera es lograda por el FEM.

La Figura 4.4 y la tabla 4.2 muestran los errores y el orden de convergencia alcanzado.
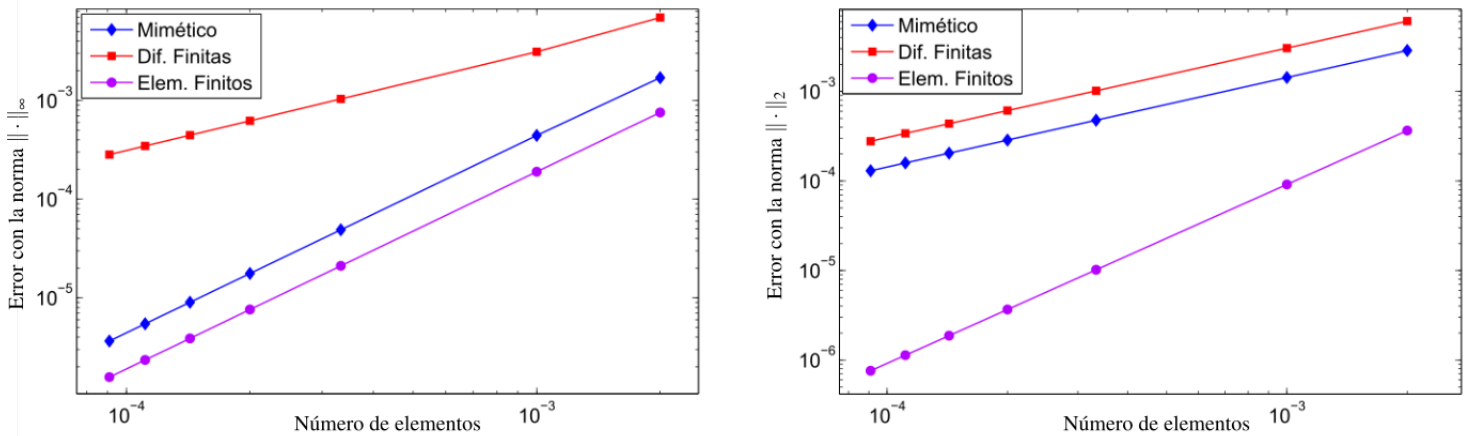

Figura 4.4: Experimentación \#2: Errores numéricos en la norma $\|\cdot\|_{\infty}$ (izquierda) y la norma $\|\cdot\|_{2}($ derecha).

En norma del máximo, el método FDM pierde el orden 2 de convergencia de los esquemas usados para su construcción. Este fenómeno es debido a que los coeficientes de difusión y convectivo dependen de la variable espacial (no constantes). El MIM y FEM mantienen el orden 2 de convergencia con FEM, superando ligeramente en precisión a MIM. En norma $L_{2}$, se repite el comportamiento del ejemplo anterior: orden dos para FEM y orden uno para FDM y MIM.

\begin{tabular}{|c|ccc|ccc|}
\hline \multirow{2}{*}{$\begin{array}{c}\# \text { de } \\
\text { Elementos }\end{array}$} & \multicolumn{3}{|c|}{ Norma $\|\cdot\|_{\infty}$} & \multicolumn{3}{c|}{ Norma $\left\|^{*}\right\|_{2}$} \\
\cline { 2 - 7 } & FDM & MIM & FEM & FDM & MIM & FEM \\
\hline 1000 & 1.154080 & 1.942273 & 1.993329 & 1.004321 & 1.003528 & 1.996938 \\
3000 & 1.000175 & 2.004354 & 1.998233 & 0.998419 & 1.000636 & 1.998873 \\
5000 & 1.000073 & 1.994971 & 1.999533 & 0.998652 & 1.000109 & 1.999803 \\
7000 & 1.000047 & 2.000917 & 1.999993 & 0.999021 & 1.000045 & 1.999919 \\
9000 & 1.000035 & 2.000485 & 1.999783 & 0.999236 & 1.000025 & 1.999950 \\
11000 & 1.000028 & 1.998615 & 2.000046 & 0.999375 & 1.000016 & 1.999978 \\
\hline
\end{tabular}

Tabla 4.2: Experimentación \#2: Orden de convergencia en Norma $\|\cdot\|_{\infty}$ y Norma $\|\cdot\|_{2}$.

4.3. Experimentación \#3. Se resuelve el problema modelo (2.1)-(2.2) para un coeficiente de difusión constante $k=1,052$, velocidad convectiva $\nu=-110,5$, y un término fuente $f \equiv 0$. La solución analítica 
queda dada por $u(x)=\left(1-e^{-\lambda x}\right) /\left(1-e^{-\lambda}\right)$, con $\lambda=\nu / k$. En la experimentación numérica se toman inicialmente condiciones de contorno Dirichlet:

$$
u(0)=0, \quad u(1)=1
$$

y, posteriormente, condiciones Robin:

$$
\begin{aligned}
& u(0)+u^{\prime}(0)=\lambda /\left(1-e^{-\lambda}\right), \\
& u(1)+u^{\prime}(1)=1+\lambda e^{-\lambda} /\left(1-e^{-\lambda}\right)
\end{aligned}
$$

Para esta configuración del problema junto a condiciones de contorno Dirichlet, es altamente conocido y fácil de probar, los métodos FDM y FEM resultan equivalentes y presentan oscilaciones cuando el número local de Péclet $|\nu| h /(2 k) \gg 1$. La forma más simple de superar este fenómeno oscilatorio (físicamente no correcto) es hacer $h$ suficientemente pequeño o añadiendo un término de difusividad artificial al problema. Debe quedar claro, que en los casos en que el número local de Péclet es igual o mucho menor que 1 estos métodos no presentan oscilaciones, contrario a lo que erróneamente se afirma en [18].

La Figura 4.5 muestra las soluciones obtenidas para 50, 80 y 200 elementos en la malla. Resulta evidente que la solución del esquema mimético también presenta las oscilaciones, y para este caso necesita más nodos que FDM y FEM para evitas las oscilaciones y lograr la convergencia a la solución analítica.
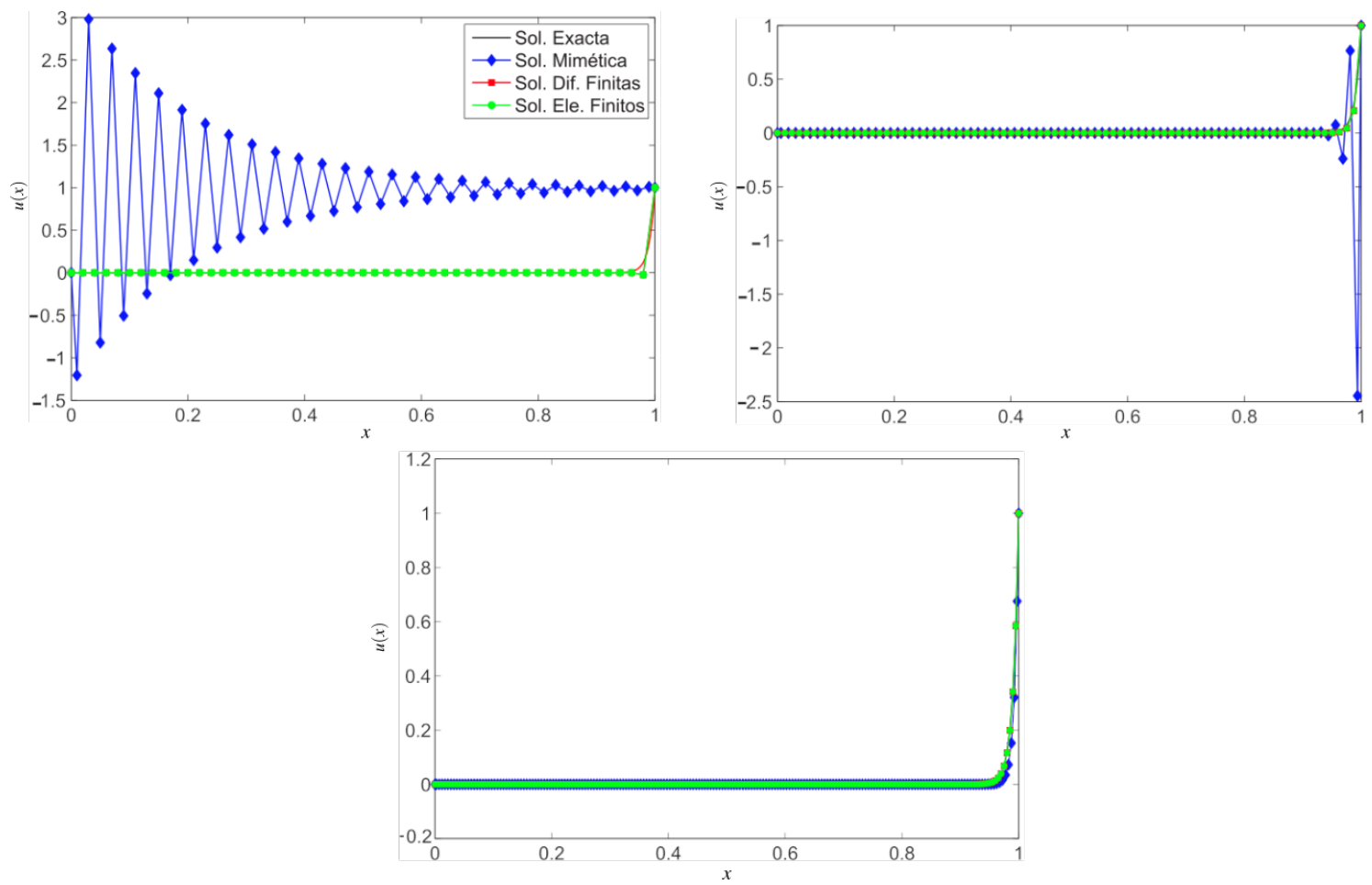

Figura 4.5: Experimentación \#3: Soluciones aproximadas y exactas con una malla de 50 nodos (arriba a la izquierda), con una malla de 80 nodos (arriba a la derecha) y una malla de 200 nodos (abajo).

La Figura 4.6 y Cuadro 4.3 muestran los errores y el orden de convergencia alcanzado para el caso de condiciones de contorno Dirichlet. 

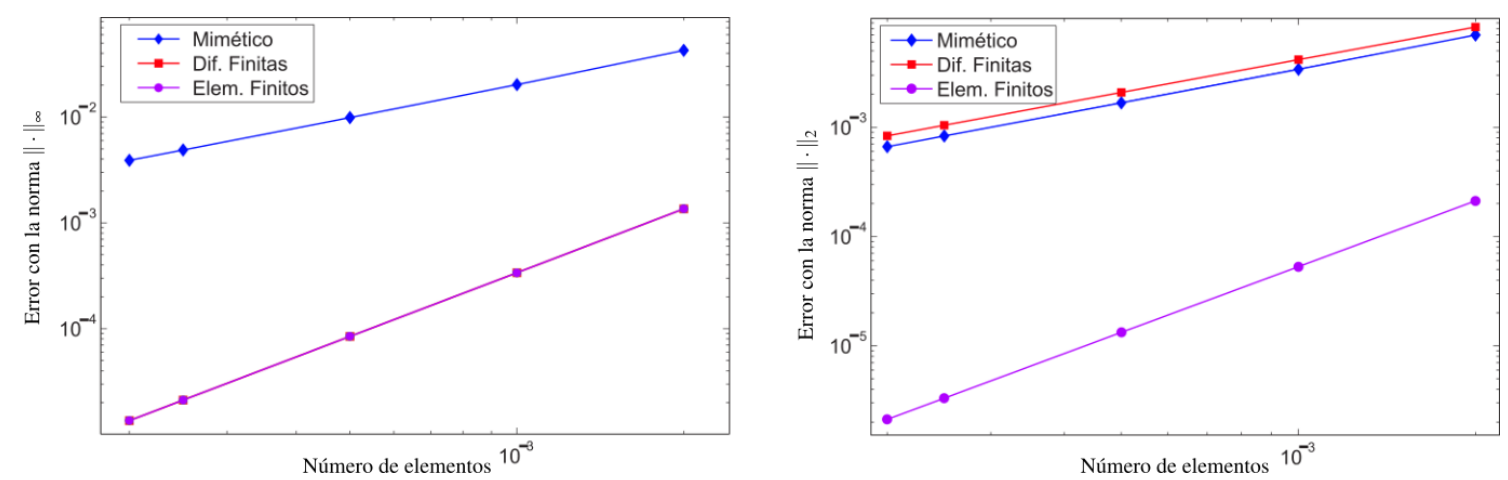

Figura 4.6: Experimentación \#2: Errores numéricos en la norma $\|\cdot\|_{\infty}$ (izquierda) y la norma $\|\cdot\|_{2}$ (derecha).

\begin{tabular}{|c|ccc|ccc|}
\hline \multirow{2}{*}{$\begin{array}{c}\# \text { de } \\
\text { Elementos }\end{array}$} & \multicolumn{3}{|c|}{ Norma $\|\cdot\|_{\infty}$} & \multicolumn{3}{c|}{ Norma $\|\cdot\|_{2}$} \\
\cline { 2 - 7 } & FDM & MIM & FEM & FDM & MIM & FEM \\
\hline 1000 & 2.005097 & 1.078854 & 2.005097 & 0.990024 & 1.042730 & 1.998561 \\
2000 & 1.999493 & 1.034747 & 1.999493 & 0.995134 & 1.018963 & 1.999640 \\
4000 & 2.000324 & 1.016534 & 2.000324 & 0.997599 & 1.008977 & 1.999910 \\
5000 & 2.000267 & 1.009977 & 2.000267 & 0.998517 & 1.005461 & 1.999966 \\
\hline
\end{tabular}

Tabla 4.3: Experimentación \#3: Orden de convergencia en Norma $\|\cdot\|_{\infty}$ y Norma $\|\cdot\|_{2}$.

En norma del máximo, como ya se dijo antes, los métodos FDM y FEM resultan equivalentes, y mantienen el orden 2 de convergencia. Sin embargo, el esquema MIM pierde su orden 2 de convergencia y su precisión es más pobre que la lograda por los otros dos métodos. En norma $L_{2}$, el método FDM se une a MIM logrando solo un orden uno en su convergencia, y con un error exiguo entre los dos. En esta norma, el FEM mantiene el orden dos de convergencia y nuevamente resulta superior en su precisión.
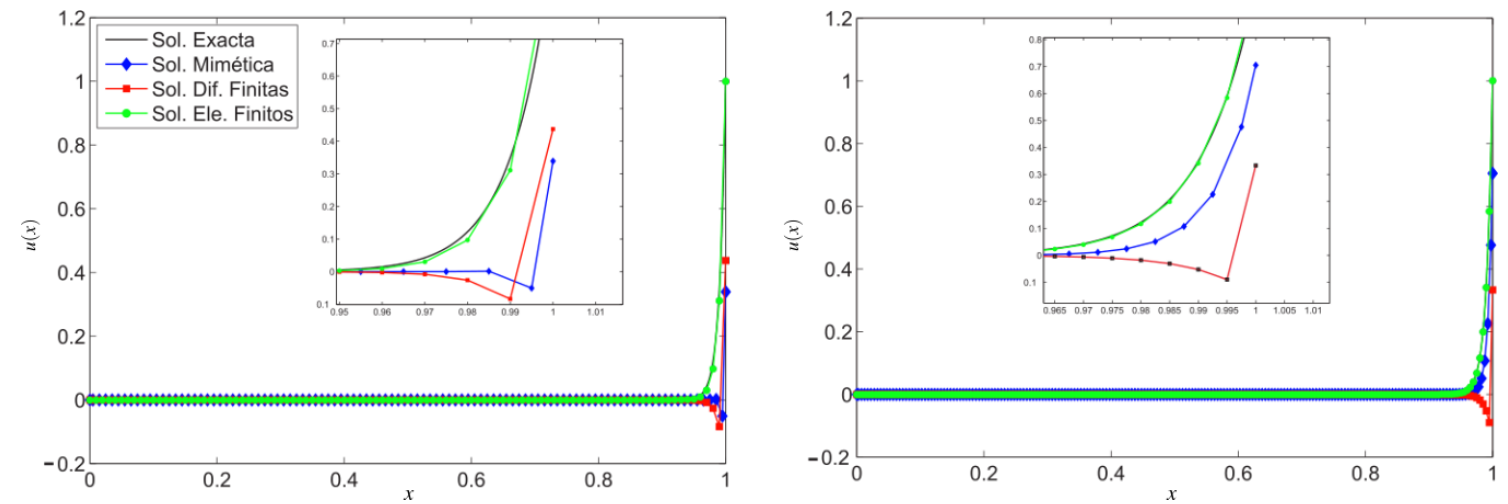

Figura 4.7: Experimentación \#3: Soluciones aproximadas y exactas en una malla de 100 nodos (izquierda) y en una malla de 200 nodos (derecha). La parte extendida de cada gráfico representa el comportamiento numérico alrededor de la frontera en $x=1$.

Para las condiciones de contorno Robin, las soluciones aproximadas son mostradas en la Figura 4.7. En la frontera $x=1$ la precisión es pobre para los métodos FDM y MIM (parte ampliada al centro de cada gráfica de la Figura 4.7). El esquema MIM llega a necesitar hasta 4 mil elementos para alcanzar la precisión que logra FEM con solo 80 elementos. EL FDM pierde precisión en esta frontera y no converge.

Los resultados de convergencia para FEM y MIM resultan equivalentes a los alcanzados para el caso de condiciones de contorno Dirichlet (orden 2 para FEM y orden 1 para MIM). Sin embargo, diferencia finitas pierde por completo su convergencia, debido a la perdida de precisión que presenta alrededor de la 
frontera $x=1$. Por último, se debe señalar que la matriz que define el sistema resultante para FEM y MIM presenta un número de condición superior a $10^{-19}$.

5. Conclusiones y comentarios finales. Basado en nuestra implementación y resultados, resulta claro, que el FEM presenta mejores resultados en precisión y convergencia que los otros dos métodos. Al mismo tiempo, MIM resulta en todo momento superior al método FDM (como ya ha sido reportado por muchas referencias). En el caso de la Experimentación \#3, donde se podría esperar superioridad del MIM, debido a su condición conservativa, tampoco logra superar a FEM. Sin querer entrar en discusión de las modificaciones apropiadas o métodos óptimos para la resolución de la Experimentación \#3, queda abierta la pregunta de qué condiciones o cambios se deben imponer al MIM para problemas altamente convectivos.

En gran medida, se ha justificado el uso de MIM ante FEM debido a la complejidad teórica de FEM. Sin embargo, la definición de los operadores discretos usados por MIM (principalmente en el caso 2D o 3D) puede resultar tan complejo en cálculo que pueden competir fácilmente con la necesidad teórica de FEM. Tal dificultad se acrecienta, si se quiere subir el orden de convergencia del método, hecho que se logra en FEM con gran facilidad. También es importante resaltar que actualmente se pueden implementar el MIM para altos ordenes, como podemos ver [19]. Y por último, actualmente, existe mucho interés en la implementación y el rendimiento del MIM en dominios con geometrías complejas, [20, 21].

Como conclusión, si se quiere abordar un problema con un método novel y se tiene a mano la discretización de los operadores, el método mimético, MIM, resultará una buena elección, por arriba de cualquier esquema de diferencias finitas. Desde la vertiente matemática, sobresale el interés de afianzar las bases teóricas del método MIM y proponer mejoras en las líneas donde el mismo aún sigue sin rigor o no han sido abordadas.

ORCID and License

Abdul Abner Lugo Jiménez https:// orcid.org/0000-0002-7667-1260

Guelvis Enrique Mata Díaz https://orcid.org/0000-0001-7147-1422

Bladismir Ruiz https: / / orcid.org/0000-0002-7737-3847

This work is licensed under the Creative Commons - Attribution 4.0 International (CC BY 4.0)

\section{Referencias}

[1] Calderón G, Lugo A. Estimación del Error y Adaptatividad en Esquemas Miméticos para Problemas de Contorno. Boletín de la Asociación Matemática Venezolana. 2015;22(2):109-124.

[2] Castillo JE, Grone RD. A Matrix Analysis Approach to Higher-Order Approximations for Divergence and Gradients Satisfying a Global Conservation Law. SIAM J Matrix Anal Appl. 2003;25(1):128-142.

[3] Hyman JM, Shashkov M. The Approximation of Boundary Conditions for Mimetic Finite Difference Methods. Computers Math Applic. 1998;36(5):79-99.

[4] Hyman JM, Shashkov M, Steinberg S. Mimetic Finite Difference Methods for Diffusion Equations. Computers Math Applic. 2002;6(3-4):333-352.

[5] Shashkov M, Steinberg S. Support-Operator Finite-Difference Algorithms for General Elliptic Problems. Journal of Computational Physics. 1995;118(1):131-151.

[6] Freites MA. Un estudio comparativo de los métodos miméticos para la ecuación estacionaria de difusión; 2004. Tesis de grado, Facultad de Ciencias, UCV.

[7] Guevara JM, Freites M, Castillo JE. A New Second Order Finite Difference Conservative Scheme. Divulgaciones Matemáticas. 2005;13(1):107-122.

[8] Li BQ. Discontinuous Finite Elements in Fluid Dynamics and Heat Transfer. 5th ed. London: Springer-Verlag; 2006.

[9] Rivière B. Discontinuous Galerkin Methods for Solving Elliptic and Parabolic Equations: Theory and Implementation. 5th ed. Philadelphia: SIAM; 2008.

[10] Cordero F, Díez P. XFEM+: una modificación de XFEM para mejorar la precisión de los flujos locales en problemas de difusión con conductividades muy distintas. Revista Internacional Métodos Numéricos para Cálculo y Diseño en Ingeniería. 2010;26(2):121-133.

[11] Arteaga J, Guevara JM. A Conservative Finite Difference Scheme for Static Diffusion Equation. Divulgaciones Matemáticas. 2008;16(1):39-54.

[12] Guevara JM. Sobre los Esquemas Miméticos de Diferencias Finitas para la Ecuación Estática de Difusión. Caracas, Venezuela: Facultad de Ciencias, UCV; 2005.

[13] Becker EB, Carey GF, Oden JT. Finite Elements: An Introduction. New Jersey 07632: Prentice-Hall, Inc.; 1981.

[14] Šolín P. Partial Differential Equations and Finite Element Method. 5th ed. New Jersey: John Wiley \& Sons, Ltd.; 2006.

[15] Calderón G, Gallo R. Introducción al Método de los Elementos Finitos: un Enfoque Matemático. Caracas, Venezuela: IVIC; 2011.

[16] Strikwerda JC. Finite Difference Schemes and Partial Differential Equations. 2nd ed. Philadelphia: SIAM, Ltd.; 2004.

[17] Céa J. Approximation variationnelle des problèmes aux limites. Annales de l'institut Fourier. 1964;14(2):345-444.

[18] Batista ED, Castillo JE. Mimetic Schemes on Non-Uniform Structured Meshes. Electronic Transactions on Numerical Analysis. 2009;34(1):152-162.

[19] Corbino J, Castillo JE. High-order mimetic finite-difference operators satisfying the extended Gauss divergence theorem. Journal of Computational and Applied Mathematics. 2020;364:112326. 
[20] Boada A, Paolini C, Castillo JE. High-order mimetic finite differences for anisotropic elliptic equations. Computers \& Fluids. 2020;213:104746.

[21] Abouali M, Castillo JE. Solving Poisson equation with Robin boundary condition on a curvilinear mesh using high order mimetic discretization methods. Mathematics and Computers in Simulation. 2017;139:23-36. 